\title{
Bose-Einstein Condensation Temperature of Dipolar Gas in Anisotropic Harmonic Trap
}

\author{
K. Glaum* \\ Fachbereich Physik, Freie Universität Berlin, Arnimallee 14, 14195 Berlin, Germany \\ A. Pelster ${ }^{\dagger}$ \\ Fachbereich Physik, Campus Duisburg, Universität Duisburg-Essen, Lotharstrasse 1, 47048 Duisburg, Germany
}

(Dated: July 29, 2021)

\begin{abstract}
We consider a dilute gas of dipole moments in an arbitrary harmonic trap and treat both the short-range, isotropic delta-interaction and the long-range, anisotropic dipole-dipole interaction perturbatively. With this we calculate the leading shift of the critical temperature with respect to that of an ideal gas as a function of the relative orientation of the dipole moments with respect to the harmonic trap axes. In particular, we determine those magic angles, where the dipolar shift of the Bose-Einstein condensation temperature vanishes. Furthermore, we show for the parameters of the ongoing ${ }^{52} \mathrm{Cr}$-experiment in Stuttgart that this dipolar shift can be enhanced by increasing the number of particles, the geometrical mean trap frequency, and the anisotropy of the trap.
\end{abstract}

PACS numbers: 03.75.Hh, 31.15.Gy, 51.30.+i

\section{INTRODUCTION}

Ultracold atomic quantum gases are many-body systems where a wide variety of macroscopic quantum phenomena is observable [1-3]. For the original Bose-Einstein condensates (BECs) of alkali atoms, it has been sufficient to describe the dominant two-particle interaction by a short-range and isotropic contact potential

$$
V_{\delta}^{(\mathrm{int})}\left(\mathbf{x}-\mathbf{x}^{\prime}\right)=\frac{4 \pi \hbar^{2} a_{s}}{M} \delta\left(\mathbf{x}-\mathbf{x}^{\prime}\right)
$$

where $M$ stands for the mass of the particles. The s-wave scattering length $a_{s}$ is tuned by using a so-called Feshbach resonance [4], where it can be varied over a broad range via an external magnetic field. Recently, a BEC has also been realized in a dipolar quantum gas of ${ }^{52} \mathrm{Cr}$ atoms [5] where the magnetic dipole moments are around six times larger than those of alkali atoms. Therefore, the physical properties of such a chromium BEC also depend on a longrange and anisotropic magnetic dipole-dipole interaction. Other many-body systems with dipolar interactions are, for instance, Rydberg atoms $[6,7]$ or atomic condensates where a strong electric field induces electric dipole moments of the order of $10^{-2}$ Debye [8]. Permanent dipole moments in heteronuclear molecules are much larger with typical values of 1 Debye, so their dipolar effects could be a few hundred times stronger than those of chromium atoms [9, 10]. Such a gas of ultracold heteronuclear molecules is produced either by sophisticated cooling and trapping techniques [11-14] or by photoassociation [15-18]. For all those systems the specific aniosotropic dipole-dipole interaction reads

$$
V_{D D}^{(\mathrm{int})}\left(\mathbf{x}-\mathbf{x}^{\prime}\right)=-\frac{\mu_{0}}{4 \pi}\left\{\frac{3\left[\mathbf{m}\left(\mathbf{x}-\mathbf{x}^{\prime}\right)\right]^{2}}{\left|\mathbf{x}-\mathbf{x}^{\prime}\right|^{5}}-\frac{\mathbf{m}^{2}}{\left|\mathbf{x}-\mathbf{x}^{\prime}\right|^{3}}\right\}+\frac{\mu_{0} m^{2}}{3} \delta\left(\mathbf{x}-\mathbf{x}^{\prime}\right),
$$

where $\mathbf{m}$ denotes the magnetic (electric) dipole moment and $\mu_{0}$ stands for the permeability constant (the reciprocal permittivity constant). Note that this dipole-dipole interaction contains, apart from the usual first term, an additional contact term, which renormalizes the divergence at the origin. The latter contribution has been overlooked in most textbooks of electrodynamics, but is taken into account in more accurate representations as, for instance, in Ref. [19]. The dipolar interaction strength can be tuned for induced dipole moments by varying the field strength and for permanent dipoles by using rotating magnetic (electric) fields [20]. Combining this rotation technique with Feshbach resonances, will allow in the near future experiments where the interaction varies from purely contact to purely dipolar. The anisotropic dipole-dipole interaction gives rise to new condensate properties as, for instance,

\footnotetext{
*Electronic address: glaum@physik.fu-berlin.de
}

${ }^{\dagger}$ Electronic address: axel.pelster@uni-due.de 


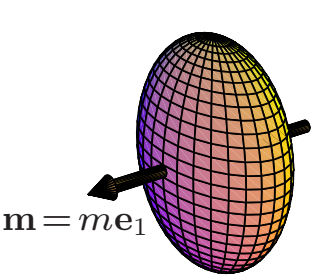

case 1

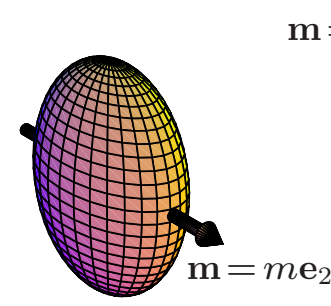

case 2

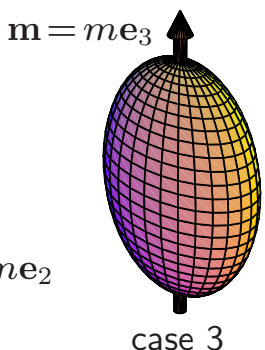

case 3

FIG. 1: Axes of magnetic dipole-dipole interaction (2) and harmonic trap (3) with frequencies $\omega_{1}>\omega_{2}>\omega_{3}$.

a characteristic anisotropic deformation of the expanding BEC which has recently been resolved experimentally in Ref. [21]. Also other interesting dipolar phenomena are accessible experimentally as, for instance, the occurrence of a Maxon-Roton in the excitation of dipolar BEC [22] or the instability of the ground state of dipolar BECs [6, 23-25].

In the present article we investigate how the critical temperature of a BEC is shifted due to the dipole-dipole interaction (2). To this end we consider a dilute gas trapped in an arbitrary harmonic potential

$$
V(\mathbf{x})=\frac{M}{2} \sum_{j=1}^{3} \omega_{j}^{2} x_{j}^{2},
$$

where $\omega_{1}, \omega_{2}, \omega_{3}$ denote the respective trap frequencies. Furthermore, we assume that, due to an additional external field, the dipole moments $\mathbf{m}$ of all constituents are oriented along one axis, i.e. they are uniformly described by $\mathbf{m}=m(\sin \alpha \cos \phi, \sin \alpha \sin \phi, \cos \alpha)$. We expect that the critical temperature depends crucially on the spherical angles $\alpha$ and $\phi$ which characterize the orientation of the dipole moments with respect to the harmonic trap axes. The temperature shift should have local extrema when the dipole moments are parallel to one of the harmonic trap axes as is illustrated in Figure 1. For instance, if the frequencies are ordered according to $\omega_{1}>\omega_{2}>\omega_{3}$, this leads to an additional interaction which is attractive along the 3 -axis, repulsive along the 1 -axis and can be both attractive or repulsive along the 2 -axis. Therefore, the particle density in the harmonic trap is increased (decreased) in case 3 (case 1) in comparison with a pure contact interaction. Thus, we expect a resulting positive (negative) shift of the critical temperature due to the magnetic dipole-dipole interaction. Furthermore, we follow Ref. [26] and suggest to determine differences of the critical temperatures in the three configurations, as then the influence of the isotropic contact interaction cancels. Consequently, those differences represent a clear signal of the anisotropic dipole-dipole interaction.

We start our detailed analysis by briefly reviewing the calculation of the Bose-Einstein condensation temperature for a dilute ideal Bose gas in Section II. Subsequently, we treat the influence of both interactions (1) and (2) in lowest order perturbatively. This is justified as the confinement of the harmonic trapping potential (3) removes critical long-wavelength fluctuations and reduces the fraction of atoms taking part in nonperturbative physics at the transition point [27]. Therefore, we determine in Section III within first-order perturbation theory how the grandcanonical free energy and, thus, the particle number equation change under the influence of an arbitrary two-particle interaction potential. Additionally, we calculate in lowest perturbative order the location of the critical point where the phase transition from a Bose gas to a BEC occurs. To this end we extract the critical chemical potential from the first-order contribution to the self-energy. This procedure is, in principle, self-consistent and corresponds to the Hartree-Fock mean-field theory. However, we determine the critical chemical potential only in the leading order and use it to obtain the critical temperture shift. Note that this provides a reliable prediction since it is only linear in the interaction strength. Due to the Ginzburg criterion critical fluctuations in the vicinity of the critial point violate the mean-field result in higher than first order of the interaction strength [28]. In Section IV we specialize our result to the model interaction potential (1) and (2) and combine it in Section V in order to derive the leading shift of the critical temperature. Furthermore, we show how it is possible to tune the strength of this dipolar shift. To this end we investigate in Section VI the magic angles where the dipolar effects vanish. Finally, we discuss in Section VII our theoretical findings for the special parameters of the ongoing chromium experiment in Stuttgart. In particular, we estimate which experimental parameters allow to enhance the differences of the critical temperatures for two of the three configurations of Figure 1. 


\section{INTERACTION-FREE CASE}

The quantum statistical properties of a dilute ideal Bose gas are determined by the grand-canonical partition function which follows from the functional integral

$$
\mathcal{Z}^{(0)}=\oint \mathcal{D} \psi^{*} \mathcal{D} \psi e^{-\mathcal{A}^{(0)}\left[\psi^{*}, \psi\right] / \hbar}
$$

Here $\oint \mathcal{D} \psi^{*} \mathcal{D} \psi$ sums over all bosonical field configurations $\psi(\mathbf{x}, \tau)$ and $\psi^{*}(\mathbf{x}, \tau)$ which are periodic in imaginary time $\tau$. Furthermore, the undisturbed euclidean action in (4) is

$$
\mathcal{A}^{(0)}\left[\psi^{*}, \psi\right]=\hbar \int_{0}^{\hbar \beta} d \tau \int_{0}^{\hbar \beta} d \tau^{\prime} \int d^{3} x \int d^{3} x^{\prime} \psi^{*}(\mathbf{x}, \tau) G^{(0)-1}\left(\mathbf{x}, \tau ; \mathbf{x}^{\prime}, \tau^{\prime}\right) \psi\left(\mathbf{x}^{\prime}, \tau^{\prime}\right)
$$

with the integral kernel

$$
G^{(0)-1}\left(\mathbf{x}, \tau ; \mathbf{x}^{\prime}, \tau^{\prime}\right)=\frac{1}{\hbar} \delta\left(\mathbf{x}-\mathbf{x}^{\prime}\right) \delta\left(\tau-\tau^{\prime}\right)\left\{\hbar \frac{\partial}{\partial \tau}-\frac{\hbar^{2}}{2 M} \mathbf{\Delta}+V(\mathbf{x})-\mu\right\},
$$

where $V(\mathbf{x})$ is the external trap potential and $\mu$ stands for the chemical potential. The grand-canonical free energy of such an ideal Bose gas is given by $\mathcal{F}^{(0)}=-\left(\ln \mathcal{Z}^{(0)}\right) / \beta$ and may be evaluated from $(4)$ as $\mathcal{F}^{(0)}=\operatorname{Tr} \ln G^{(0)-1} / \beta$. For the harmonic trap potential (3), usually the condition $\hbar \beta \tilde{\omega} \ll 1$ holds, where $\tilde{\omega}=\left(\omega_{1} \omega_{2} \omega_{3}\right)^{1 / 3}$ denotes the geometric mean of the trap frequencies. This allows to determine the grand-canonical free energy within a semiclassical treatment $[29]$

$$
\mathcal{F}^{(0)}=-\frac{1}{\beta(\hbar \beta \tilde{\omega})^{3}} \zeta_{4}\left(e^{\beta \mu}\right)
$$

where $\zeta_{a}(z)=\sum_{n=1}^{\infty} z^{n} / n^{a}$ represents the polylogarithmic function. Now we are interested in thermodynamic properties which result from a fixed average particle number $N=-\partial \mathcal{F}^{(0)} / \partial \mu$ :

$$
N=\frac{1}{(\hbar \beta \tilde{\omega})^{3}} \zeta_{3}\left(e^{\beta \mu}\right)
$$

In particular, we are interested in calculating the critical temperature where a macroscopic occupation of the ground state sets in. The critical point can be immediately read off from Eq. (8) to be $\mu_{c}^{(0)}=0$, since there the polylogarithmic function starts to diverge. However, this critical point, where the phase transition from a Bose gas to a BEC occurs, also coincides with a divergence of the correlation function according to the theory of critical phenomena [30]. To this end we determine the correlation function of an ideal Bose gas from the function integral

$$
G^{(0)}\left(\mathbf{x}, \tau ; \mathbf{x}^{\prime}, \tau^{\prime}\right)=\frac{1}{\mathcal{Z}^{(0)}} \oint \mathcal{D} \psi^{*} \mathcal{D} \psi \psi(\mathbf{x}, \tau) \psi^{*}\left(\mathbf{x}^{\prime}, \tau^{\prime}\right) e^{-\mathcal{A}^{(0)}\left[\psi^{*}, \psi\right] / \hbar} .
$$

The semiclassical result reads

$$
\begin{aligned}
G^{(0)}\left(\mathbf{x}, \tau ; \mathbf{x}^{\prime}, \tau^{\prime}\right)= & \int \frac{d^{3} p}{(2 \pi \hbar)^{3}} e^{i \mathbf{p}\left(\mathbf{x}-\mathbf{x}^{\prime}\right) / \hbar}\left\{\Theta\left(\tau-\tau^{\prime}\right) \sum_{n=0}^{\infty} e^{-\frac{1}{\hbar}\left[\frac{\mathbf{p}^{2}}{2 M}+V\left(\frac{\mathbf{x}+\mathbf{x}^{\prime}}{2}\right)-\mu\right]\left(\tau-\tau^{\prime}+n \hbar \beta\right)}\right. \\
& \left.+\Theta\left(\tau^{\prime}-\tau\right) \sum_{n=1}^{\infty} e^{-\frac{1}{\hbar}\left[\frac{\mathbf{p}^{2}}{2 M}+V\left(\frac{\mathbf{x}+\mathbf{x}^{\prime}}{2}\right)-\mu\right]\left(\tau-\tau^{\prime}+n \hbar \beta\right)}\right\}
\end{aligned}
$$

The Fourier-Matsubara transform for such a semiclassical expression is given by

$$
G^{(0)}\left(\mathbf{p}, \omega_{m} ; \mathbf{x}\right)=\int_{0}^{\hbar \beta} d \tau e^{i \omega_{m} \tau} \int d^{D} x^{\prime} e^{-i \mathbf{p} \mathbf{x}^{\prime} / \hbar} G^{(0)}\left(\mathbf{x}+\frac{\mathbf{x}^{\prime}}{2}, \tau ; \mathbf{x}-\frac{\mathbf{x}^{\prime}}{2}, 0\right),
$$

where $\omega_{m}=2 \pi m / \hbar \beta$ denotes the Matsubara frequencies. Inserting (10) in (11) yields

$$
G^{(0)}\left(\mathbf{p}, \omega_{m} ; \mathbf{x}\right)=\frac{\hbar}{-i \hbar \omega_{m}+\frac{\mathbf{p}^{2}}{2 M}+V(\mathbf{x})-\mu}
$$


which reveals explicitly that (9) represents the functional inverse of the integral kernel (6). At the critical point, where the correlation function (9) diverges, the integral kernel vanishes. Within the semiclassical approximation of the Fourier-Matsubara transform of (6) we thus obtain

$$
G^{(0)-1}\left(\mathbf{p}, \omega_{m} ; \mathbf{x}\right)=\frac{1}{\hbar}\left\{-i \hbar \omega_{m}+\frac{\mathbf{p}^{2}}{2 M}+V(\mathbf{x})-\mu\right\}=0 .
$$

This equation can only be fulfilled for vanishing momentum $\mathbf{p}=\mathbf{0}$ and Matsubara frequency $\omega_{m}=0$ at the critical chemical potential

$$
\mu_{c}^{(0)}=\min _{\mathbf{x}} V(\mathbf{x})
$$

In case of the harmonic potential (3) the minimum occurs in the center of the trap. Thus, we reproduce the critical chemical potential $\mu_{c}^{(0)}=0$, which corresponds to the semiclassical value of the ground-state energy. Inserting this into Eq. (8), we obtain for the transition temperature for an interaction-free Bose gas

$$
T_{c}^{(0)}=\frac{\hbar \tilde{\omega}}{k_{B}}\left[\frac{N}{\zeta(3)}\right]^{1 / 3},
$$

where $\zeta(a)=\sum_{n=1}^{\infty} 1 / n^{a}$ is the so-called Riemann zeta function. Note that in more complicated traps, which arise, for instance, when the Bose gas is rotated, the potential minimum could occur far away from the origin, thus resulting in a non-vanishing critical chemical potential [31].

From the critical temperature (15) we read off that $\hbar \beta_{c}^{(0)} \tilde{\omega} \sim N^{-1 / 3}$, i.e. the condition for the semiclassical treatment is fulfilled within the thermodynamic limit $N \rightarrow \infty$. For a finite number of particles $N$, it is necessary to go beyond the semiclassical approximation and to take into account quantum corrections in a systematic way [32-35]. In lowest order this finite-size effect in harmonic traps follows from identifying $\mu_{c}^{(0)}$ not with the semiclassical value of the ground-state energy but with its quantum mechanical value $E_{G}=3 \hbar \bar{\omega} / 2$, where $\bar{\omega}=\left(\omega_{1}+\omega_{2}+\omega_{3}\right) / 3$ denotes the arithmetic mean of the trap frequencies. Evaluating the polylogarithmic function in (8) perturbatively up to the leading order, we obtain for the critical temperature the finite-size effect

$$
\left(\frac{\Delta T_{c}}{T_{c}^{(0)}}\right)_{F S}=-\frac{\zeta(2) \bar{\omega}}{2 \zeta^{2 / 3}(3) \tilde{\omega} N^{1 / 3}}
$$

The reduction of the critical temperature of an ideal gas decreases with increasing the particle number $N$.

\section{FIRST-ORDER PERTURBATION THEORY}

In this section we investigate the influence of an arbitrary weak 2-particle interaction upon the thermodynamic properties of dilute Bose gases within Feynman's diagrammatic technique of many-body theory [36-41].

\section{A. Feynman Rules}

We start with defining the grand-canonical partition function of the full problem

$$
\mathcal{Z}=\oint \mathcal{D} \psi^{*} \mathcal{D} \psi e^{-\left(\mathcal{A}^{(0)}\left[\psi^{*}, \psi\right]+\mathcal{A}^{(\mathrm{int})}\left[\psi^{*}, \psi\right]\right) / \hbar}
$$

and the associated correlation function

$$
G\left(\mathbf{x}, \tau ; \mathbf{x}^{\prime}, \tau^{\prime}\right)=\frac{1}{\mathcal{Z}} \oint \mathcal{D} \psi^{*} \mathcal{D} \psi \psi(\mathbf{x}, \tau) \psi^{*}\left(\mathbf{x}^{\prime}, \tau^{\prime}\right) e^{-\left(\mathcal{A}^{(0)}\left[\psi^{*}, \psi\right]+\mathcal{A}^{(\mathrm{int})}\left[\psi^{*}, \psi\right]\right) / \hbar} .
$$

In addition to the interaction-free contribution to the action (5) we have also to take into account the interaction contribution. Due to the diluteness of the Bose gas we can restrict ourselves to the effects of a two-particle interaction $V^{(\text {int })}\left(\mathbf{x}-\mathbf{x}^{\prime}\right)$ :

$$
\mathcal{A}^{(\mathrm{int})}\left[\psi^{*}, \psi\right]=\frac{1}{2} \int_{0}^{\hbar \beta} d \tau \int d^{3} x \int d^{3} x^{\prime} V^{(\mathrm{int})}\left(\mathbf{x}-\mathbf{x}^{\prime}\right) \psi^{*}(\mathbf{x}, \tau) \psi^{*}\left(\mathbf{x}^{\prime}, \tau\right) \psi(\mathbf{x}, \tau) \psi\left(\mathbf{x}^{\prime}, \tau\right) .
$$


By expanding the functional interals (17), (18) in powers of $V^{(i n t)}\left(\mathbf{x}-\mathbf{x}^{\prime}\right)$, we obtain expressions which consist of interaction-free expectation values. These are evaluated with the help of Wick's rule as a sum of Feynman integrals, which are pictured as diagrams constructed from lines and vertices. A straight line with an arrow represents the interaction-free correlation function (9):

$$
\mathbf{x}, \tau \longleftarrow \mathbf{x}^{\prime}, \tau^{\prime} \equiv G^{(0)}\left(\mathbf{x}, \tau ; \mathbf{x}^{\prime}, \tau^{\prime}\right) .
$$

Furthermore, spatio-temporal integrals over the two-particle interaction potential are pictured by two vertices connected by a dashed line

$$
\text { x. }{ }^{\prime} \mathbf{x}^{\prime} \equiv-\frac{1}{\hbar} \int_{0}^{\hbar \beta} d \tau \int d^{3} x \int d^{3} x^{\prime} V^{(\mathrm{int})}\left(\mathbf{x}-\mathbf{x}^{\prime}\right)
$$

In the following we apply these Feynman rules in order to determine the grand-canonical free energy and the selfenergy within first-order perturbation theory for the harmonic trap potential (3) and an arbitrary interaction potential $V^{(\text {int })}\left(\mathbf{x}-\mathbf{x}^{\prime}\right)$.

\section{B. Grand-Canonical Free Energy}

Up to the first order in the two-particle interaction the grand-canonical free energy $\mathcal{F}=-(\ln \mathcal{Z}) / \beta$ reads with the diagrammatic rules mentioned above

$$
\mathcal{F}=\mathcal{F}^{(0)}-\frac{1}{\beta}\left\{\frac{1}{2} \bigcirc+\frac{1}{2} \circlearrowleft+\ldots\right\}
$$

By passing we note that those two and all higher-order connected vacuum diagrams follow together with their proper weights from solving a graphical recursion relation [42]. The first term in (22) is the interaction-free contribution to the grand-canonical free energy (7). The second and third term in (22) are called the direct or Hartree-like and exchange or Fock-like vacuum diagrams, respectively, which correspond to the following analytical expressions:

$$
\begin{aligned}
& \mathcal{F}^{(\mathrm{D})}=\frac{1}{2 \hbar \beta} \int_{0}^{\hbar \beta} d \tau \int d^{3} x \int d^{3} x^{\prime} V^{(\mathrm{int})}\left(\mathbf{x}-\mathbf{x}^{\prime}\right) G^{(0)}(\mathbf{x}, \tau ; \mathbf{x}, \tau) G^{(0)}\left(\mathbf{x}^{\prime}, \tau ; \mathbf{x}^{\prime}, \tau\right) \\
& \mathcal{F}^{(\mathrm{E})}=\frac{1}{2 \hbar \beta} \int_{0}^{\hbar \beta} d \tau \int d^{3} x \int d^{3} x^{\prime} V^{(\mathrm{int})}\left(\mathbf{x}-\mathbf{x}^{\prime}\right) G^{(0)}\left(\mathbf{x}, \tau ; \mathbf{x}^{\prime}, \tau\right) G^{(0)}\left(\mathbf{x}^{\prime}, \tau ; \mathbf{x}, \tau\right) .
\end{aligned}
$$

Both contain the interaction-free correlation function (10) with equal imaginary times. In order to guarantee the normal operator ordering within the functional integral formalism, this equal-time correlation function $G^{(0)}\left(\mathbf{x}, \tau ; \mathbf{x}^{\prime}, \tau\right)$ must be interpreted as $G^{(0)}\left(\mathbf{x}, \tau ; \mathbf{x}^{\prime}, \tau^{+}\right)[38,39]$. Here we have introduced $\tau^{+}$as an imaginary time which is infinitesimally later than $\tau$. With this and a Fourier transformation of the interaction potential we obtain for the harmonic trap potential (3):

$$
\begin{aligned}
& \mathcal{F}^{(\mathrm{D})}=\frac{1}{2(\hbar \beta \tilde{\omega})^{6}} \sum_{n=1}^{\infty} \sum_{n^{\prime}=1}^{\infty} \frac{e^{\left(n+n^{\prime}\right) \beta \mu}}{n^{3} n^{\prime 3}} \int \frac{d^{3} q}{(2 \pi \hbar)^{3}} V^{(\mathrm{int})}(\mathbf{q}) \exp \left\{-\sum_{j=1}^{3} \frac{\left(n+n^{\prime}\right) q_{j}^{2}}{2 \hbar^{2} \beta M n n^{\prime} \omega_{j}^{2}}\right\}, \\
& \mathcal{F}^{(\mathrm{E})}=\frac{1}{2(\hbar \beta \tilde{\omega})^{3}} \sum_{n=1}^{\infty} \sum_{n^{\prime}=1}^{\infty} \frac{e^{\left(n+n^{\prime}\right) \beta \mu}}{\left(n+n^{\prime}\right)^{3}} \int \frac{d^{3} q}{(2 \pi \hbar)^{3}} V^{(\mathrm{int})}(\mathbf{q}) \exp \left\{-\frac{\beta n n^{\prime} \mathbf{q}^{2}}{2 M\left(n+n^{\prime}\right)}\right\} .
\end{aligned}
$$

\section{Self-Energy}

In order to determine the location of the phase transition from a Bose gas to a BEC, we follow the physical notion elucidated in the ideal case of the previous section and investigate when the correlation function (18) diverges, i.e., when its functional inverse $G^{-1}\left(\mathbf{x}, \tau ; \mathbf{x}^{\prime}, \tau^{\prime}\right)$ vanishes. Due to the two-particle interaction, it decomposes into the integral kernel (6) and the self-energy $\Sigma\left(\mathbf{x}, \tau ; \mathbf{x}^{\prime}, \tau^{\prime}\right)$ according to

$$
G^{-1}\left(\mathbf{x}, \tau ; \mathbf{x}^{\prime}, \tau^{\prime}\right)=G^{(0)-1}\left(\mathbf{x}, \tau ; \mathbf{x}^{\prime}, \tau^{\prime}\right)-\Sigma\left(\mathbf{x}, \tau ; \mathbf{x}^{\prime}, \tau^{\prime}\right),
$$


which is equivalent to the Dyson equation [36-41]

$$
\begin{aligned}
G\left(\mathbf{x}, \tau ; \mathbf{x}^{\prime}, \tau^{\prime}\right)= & G^{(0)}\left(\mathbf{x}, \tau ; \mathbf{x}^{\prime}, \tau^{\prime}\right) \\
& +\int d^{3} x^{\prime \prime} \int d^{3} x^{\prime \prime \prime} \int_{0}^{\hbar \beta} d \tau^{\prime \prime} \int_{0}^{\hbar \beta} d \tau^{\prime \prime \prime} G\left(\mathbf{x}, \tau ; \mathbf{x}^{\prime \prime}, \tau^{\prime \prime}\right) \Sigma\left(\mathbf{x}^{\prime \prime}, \tau^{\prime \prime} ; \mathbf{x}^{\prime \prime \prime}, \tau^{\prime \prime \prime}\right) G^{(0)}\left(\mathbf{x}^{\prime \prime \prime}, \tau^{\prime \prime \prime} ; \mathbf{x}^{\prime}, \tau^{\prime}\right) .
\end{aligned}
$$

Therefore, a perturbative evaluation of the self-energy yields via (28) self-consistent interaction corrections to the free correlation function (9). In lowest perturbative order the self-energy is defined by the diagrams

$$
\Sigma\left(\mathbf{x}, \tau ; \mathbf{x}^{\prime}, \tau^{\prime}\right)=\bigodot_{\mathbf{x}, \tau \ldots \mathbf{x}^{\prime}, \tau^{\prime}}+\underset{\mathbf{x}, \tau \ldots \mathbf{x}^{\prime}, \tau^{\prime}}{+\ldots},
$$

which correspond to the analytical expressions

$$
\begin{aligned}
& \Sigma^{(\mathrm{D})}\left(\mathbf{x}, \tau ; \mathbf{x}^{\prime}, \tau^{\prime}\right)=\frac{-1}{\hbar} \delta\left(\tau-\tau^{\prime}\right) \delta\left(\mathbf{x}-\mathbf{x}^{\prime}\right) \int d^{3} x^{\prime \prime} V^{(\mathrm{int})}\left(\mathbf{x}-\mathbf{x}^{\prime \prime}\right) G^{(0)}\left(\mathbf{x}^{\prime \prime}, \tau ; \mathbf{x}^{\prime \prime}, \tau\right), \\
& \Sigma^{(\mathrm{E})}\left(\mathbf{x}, \tau ; \mathbf{x}^{\prime}, \tau^{\prime}\right)=\frac{-1}{\hbar} \delta\left(\tau-\tau^{\prime}\right) V^{(\mathrm{int})}\left(\mathbf{x}-\mathbf{x}^{\prime}\right) G^{(0)}\left(\mathbf{x}, \tau ; \mathbf{x}^{\prime}, \tau\right) .
\end{aligned}
$$

Note that the diagrams (29) follow from amputating a line in the connected vacuum diagrams of the grand-canonical free energy $[43,44]$. Thus, using the first-order perturbative approximation (29) for the self-energy corresponds via the Dyson equation (28) to a nonperturbative Hartree-Fock mean-field theory for the correlation function.

To find the zero of (27), we consider its Fourier-Matsubara transform. Together with (6) this leads to the equation

$$
\frac{1}{\hbar}\left\{-i \hbar \omega_{m}+\frac{\mathbf{p}^{2}}{2 M}+V(\mathbf{x})-\mu\right\}-\Sigma\left(\mathbf{p}, \omega_{m} ; \mathbf{x}\right)=0
$$

where the Fourier-Matsubara transform of the self-energy follows in analogy to Eq. (11) from

$$
\Sigma\left(\mathbf{p}, \omega_{m} ; \mathbf{x}\right)=\int_{0}^{\hbar \beta} d \tau e^{i \omega_{m} \tau} \int d^{D} x^{\prime} e^{-i \mathbf{p x ^ { \prime }} / \hbar} \Sigma\left(\mathbf{x}+\frac{\mathbf{x}^{\prime}}{2}, \tau ; \mathbf{x}-\frac{\mathbf{x}^{\prime}}{2}, 0\right)
$$

Eq. (32) is fulfilled up to the lowest perturbative order at vanishing momentum $\mathbf{p}=\mathbf{0}$ and Matsubara frequency $\omega_{m}=0$ as in the previous section. Furthermore, we conclude that the additional contributions (30), (31) in (32) do not change the location $\mathbf{x}_{\min }=\mathbf{0}$ of the potential minimum. Therefore, the critical chemical potential is given up to first order by $\mu_{c}=-\hbar \Sigma(\mathbf{0}, 0 ; \mathbf{0})$. Identifying the direct and the exchange contribution with $\mu_{c}^{(\mathrm{D})}=-\hbar \Sigma^{(\mathrm{D})}(\mathbf{0}, 0 ; \mathbf{0})$ and $\mu_{c}^{(\mathrm{E})}=-\hbar \Sigma^{(\mathrm{E})}(\mathbf{0}, 0 ; \mathbf{0})$, we obtain $\mu_{c}=\mu_{c}^{(\mathrm{D})}+\mu_{c}^{(\mathrm{E})}+\ldots$, where the respective terms read according to (10)

$$
\begin{aligned}
& \mu_{c}^{(\mathrm{D})}=\frac{1}{(\hbar \beta \tilde{\omega})^{3}} \sum_{n=1}^{\infty} \frac{1}{n^{3}} \int \frac{d^{3} q}{(2 \pi \hbar)^{3}} V^{(\mathrm{int})}(\mathbf{q}) \exp \left\{-\sum_{j=1}^{3} \frac{q_{j}^{2}}{2 \hbar^{2} \beta M n \omega_{j}^{2}}\right\}, \\
& \mu_{c}^{(\mathrm{E})}=\sum_{n=1}^{\infty} \int \frac{d^{3} q}{(2 \pi \hbar)^{3}} V^{(\mathrm{int})}(\mathbf{q}) \exp \left\{-\sum_{j=1}^{3} \frac{\beta n q_{j}^{2}}{2 M}\right\} .
\end{aligned}
$$

\section{MODEL INTERACTION}

In this section we specialize our general formulas for the two-particle interaction between dipolar bosons which contains both the contact interaction (1) and the dipole-dipole interaction (2). At first, we briefly discuss whether applying this model interaction is physically reasonable. Recently, it has been suggested in Refs. [45, 46] that the $s$-wave scattering length $a_{s}$ could strongly depend on the dipole moment. However, there it has also been shown that for dipolar interaction strengths, which are not larger than the s-wave scattering strength, the latter is only rescaled by a moderate factor and remains positive. For the calculations in the present work we assume that this condition is fulfilled. Furthermore, we mention that the contact interaction (1) represents an effective pseudopotential, see e.g. Refs. [36, 47]. An analogous pseudopotential for anisotropic interactions has recently been introduced in 
Refs. $[48,49]$ which contains, apart from the usual dipole-dipole interaction (2) in the s-d scattering channel, also another part from the d-s scattering channel. The contribution of the latter turns out to be nonlocal in momentum space and is therefore difficult to handle. Here we assume that we are not in the vicinity of any dipolar shape resonance and, hence, adopt the anisotropic interaction in the original form (2). Since there exists up to now no experimental evidence that a scattering in the d-s channel is relevant, our model seems to be valid for all current experimental situations.

\section{A. Fourier Representation}

Now we combine both interactions (1), (2) and write our model interaction in the following form:

$$
V^{(\mathrm{int})}\left(\mathbf{x}-\mathbf{x}^{\prime}\right)=\frac{4 \pi \hbar^{2} a}{M} \delta\left(\mathbf{x}-\mathbf{x}^{\prime}\right)-\frac{\mu_{0}}{4 \pi}\left\{\frac{3\left[\mathbf{m}\left(\mathbf{x}-\mathbf{x}^{\prime}\right)\right]^{2}}{\left|\mathbf{x}-\mathbf{x}^{\prime}\right|^{5}}-\frac{\mathbf{m}^{2}}{\left|\mathbf{x}-\mathbf{x}^{\prime}\right|^{3}}\right\} .
$$

Here we used the effective scattering length

$$
a \equiv a_{s}+\frac{\mu_{0} m^{2} M}{12 \pi \hbar^{2}}
$$

for describing the strength of the contact interaction. Note that both terms in the effective scattering length (37), i.e. the original s-wave scattering length $a_{s}$ and the magnetic dipole contribution, are physically inseparable. Therefore, we have to identify (37) with the experimentally measurable scattering length of atoms with magnetic dipole moments. In the following we will use the dimensionless parameter

$$
\epsilon_{D D}=\frac{\mu_{0} m^{2} M}{12 \pi \hbar^{2} a}
$$

which is a measure of the strength of the dipole-dipole interaction relative to the effective scattering energy. In the limit of a vanishing scattering length $a_{s} \rightarrow 0$ the model interaction (36) describes the pure dipolar interaction (2). This corresponds to the value $\epsilon_{D D}=1$, which plays a special role by investigating the stability of a dipolar BEC at zero temperature within the Thomas-Fermi approximation [24, 25]. Under certain conditions one expects at this dipole interaction strength that a cloud of dipolar particles collapses into a thin wire along the magnetization direction. In contrast, the value $\epsilon_{D D} \rightarrow \infty$ is only achieved in the limit $a_{s} \rightarrow-\mu_{0} m^{2} M / 12 \pi \hbar^{2}$ which does not correspond to any distinguished situation. Furthermore, if we consider the limit of a vanishing dipole moment $m \rightarrow 0$, the model interaction reduces to the pure contact interaction (1).

Now we determine the Fourier representation of our model interaction (36). The Fourier transform of the contact interaction is simply a constant. The Fourier transformation of the anisotropic term has to be evaluated with special care, as the corresponding Fourier integral is UV-divergent. One possibility to regularize this singularity is to introduce an UV cut-off distance which physically takes into account that the atoms cannot overlap [9]. Thus, the Fourier transform is determined for a finite UV cut-off distance which is, finally, allowed to go to zero. Using spherical coordinates, where the dipole moment is described by $\mathbf{m}=m(\sin \alpha \cos \phi, \sin \alpha \sin \phi, \cos \alpha)$, we obtain the following Fourier transform:

$$
\begin{aligned}
V^{(\mathrm{int})}(\mathbf{q})= & \frac{4 \pi \hbar^{2} a}{M}-\frac{\mu_{0} m^{2}}{3}+\frac{\mu_{0} m^{2}}{\mathbf{q}^{2}}\left[\sin ^{2} \alpha\left(\cos ^{2} \phi q_{1}^{2}+\sin ^{2} \phi q_{2}^{2}\right)+\cos ^{2} \alpha q_{3}^{2}\right. \\
& \left.+2 \sin \alpha \cos \alpha\left(\cos \phi q_{1} q_{3}+\sin \phi q_{2} q_{3}\right)+2 \sin ^{2} \alpha \sin \phi \cos \phi q_{1} q_{2}\right] .
\end{aligned}
$$

Another approach for obtaining this result (39) has been explored in quantum electrodynamics in the context of calculating the transverse delta function with a regularization function [50]. Note that the result (39) also follows from applying the distributional identity [25]

$$
\frac{3 x_{i} x_{j}-\delta_{i j} \mathbf{x}^{2}}{|\mathbf{x}|^{5}}=\frac{4 \pi}{3} \delta_{i j} \delta(\mathbf{x})+\frac{\partial^{2}}{\partial x_{i} \partial x_{j}} \frac{1}{|\mathbf{x}|}
$$

which can be regarded as defining the dipole-dipole interaction as second partial derivatives of the Coulomb potential. 


\section{B. Evaluation of Integrals}

Now we evaluate the influence of the interaction (39) upon the thermodynamic quantities of interest. To this end we remark that the remaining integrals (25), (26) and (34), (35) are of the form

$$
I^{(\mathrm{int})}\left(\frac{a_{1}}{a_{2}}, \frac{a_{1}}{a_{3}}\right)=-\frac{3}{\mu_{0} m^{2} a_{1} a_{2} a_{3}} \int_{-\infty}^{\infty} \frac{d q_{1}}{\sqrt{\pi}} \int_{-\infty}^{\infty} \frac{d q_{2}}{\sqrt{\pi}} \int_{-\infty}^{\infty} \frac{d q_{3}}{\sqrt{\pi}} V^{(\mathrm{int})}(\mathbf{q}) \exp \left(-\frac{q_{1}^{2}}{a_{1}^{2}}-\frac{q_{2}^{2}}{a_{2}^{2}}-\frac{q_{3}^{2}}{a_{3}^{2}}\right) .
$$

In order to calculate this quantity, we define the integrals

$$
I^{(j)}\left(\frac{a_{1}}{a_{2}}, \frac{a_{1}}{a_{3}}\right)=\frac{3}{a_{1} a_{2} a_{3}} \int_{-\infty}^{\infty} \frac{d q_{1}}{\sqrt{\pi}} \int_{-\infty}^{\infty} \frac{d q_{2}}{\sqrt{\pi}} \int_{-\infty}^{\infty} \frac{d q_{3}}{\sqrt{\pi}}\left(\frac{1}{3}-\frac{1}{3 \epsilon_{D D}}-\frac{q_{j}^{2}}{\mathbf{q}^{2}}\right) \exp \left(-\frac{q_{1}^{2}}{a_{1}^{2}}-\frac{q_{2}^{2}}{a_{2}^{2}}-\frac{q_{3}^{2}}{a_{3}^{2}}\right)
$$

which occur for the configurations $j=1,2$, and 3 of Figure 1 , respectively. Due to the symmetry of its integrand, the full problem (41) is then solved by

$$
I^{(\mathrm{int})}\left(\frac{a_{1}}{a_{2}}, \frac{a_{1}}{a_{3}}\right)=\sin ^{2} \alpha \cos ^{2} \phi I^{(1)}\left(\frac{a_{1}}{a_{2}}, \frac{a_{1}}{a_{3}}\right)+\sin ^{2} \alpha \sin ^{2} \phi I^{(2)}\left(\frac{a_{1}}{a_{2}}, \frac{a_{1}}{a_{3}}\right)+\cos ^{2} \alpha I^{(3)}\left(\frac{a_{1}}{a_{2}}, \frac{a_{1}}{a_{3}}\right) .
$$

From this representation we obtain immediately that $I^{\text {(int) }}\left(a_{1} / a_{2}, a_{1} / a_{3}\right)$ is extremal for arbitrary parameters $a_{1}, a_{2}$ and $a_{3}$ only if

$$
\left\{\begin{array}{lll}
\alpha=\pi / 2, & \phi=0, \pi: & I^{(\mathrm{int})}\left(\frac{a_{1}}{a_{2}}, \frac{a_{1}}{a_{3}}\right)=I^{(1)}\left(\frac{a_{1}}{a_{2}}, \frac{a_{1}}{a_{3}}\right), \\
\alpha=\pi / 2, & \phi=\pi / 2,3 \pi / 2: & I^{(\mathrm{int})}\left(\frac{a_{1}}{a_{2}}, \frac{a_{1}}{a_{3}}\right)=I^{(2)}\left(\frac{a_{1}}{a_{2}}, \frac{a_{1}}{a_{3}}\right), \\
\alpha=0, & \phi-\text { arbitrary : } & I^{(\mathrm{int})}\left(\frac{a_{1}}{a_{2}}, \frac{a_{1}}{a_{3}}\right)=I^{(2)}\left(\frac{a_{1}}{a_{2}}, \frac{a_{1}}{a_{3}}\right) .
\end{array}\right.
$$

Whether these extrema lead to local maxima or minima of $I^{(\mathrm{int})}$ depends on the concrete values of the coefficients $I^{(1)}, I^{(2)}$ and $I^{(3)}$. To calculate them we mention the properties

$$
I^{(2)}\left(\frac{a_{1}}{a_{2}}, \frac{a_{1}}{a_{3}}\right)=I^{(1)}\left(\frac{a_{2}}{a_{3}}, \frac{a_{2}}{a_{1}}\right), \quad I^{(3)}\left(\frac{a_{1}}{a_{2}}, \frac{a_{1}}{a_{3}}\right)=I^{(1)}\left(\frac{a_{3}}{a_{1}}, \frac{a_{3}}{a_{2}}\right),
$$

which simply follow from definition (42). Consequently, we only need to determine one of the three integrals, for instance, that for $j=1$. Applying the Schwinger proper-time representation $1 / \mathbf{q}^{2}=\int_{0}^{\infty} d \tau e^{-\mathbf{q}^{2} \tau}[30]$, we arrive at the expression

$$
I^{(1)}\left(\frac{a_{1}}{a_{2}}, \frac{a_{1}}{a_{3}}\right)=1-\frac{1}{\epsilon_{D D}}-\frac{3}{2 a_{1} a_{2} a_{3}} \int_{0}^{\infty} d \tau \frac{1}{\left(\tau+1 / a_{1}^{2}\right)^{3 / 2}\left(\tau+1 / a_{2}^{2}\right)^{1 / 2}\left(\tau+1 / a_{3}^{2}\right)^{1 / 2}} .
$$

With the help of substitutions the remaining integral is reduced to incomplete elliptic functions of the first and second kind $[51,(8.111)]$

$$
F(\phi, k)=\int_{0}^{\sin \phi} d x \frac{1}{\left(1-x^{2}\right)^{1 / 2}\left(1-k^{2} x^{2}\right)^{1 / 2}} \quad, \quad E(\phi, k)=\int_{0}^{\sin \phi} d x \frac{\left(1-k^{2} x^{2}\right)^{1 / 2}}{\left(1-x^{2}\right)^{1 / 2}} .
$$

We present the result in the following form:

$$
I^{(1)}\left(\frac{a_{1}}{a_{2}}, \frac{a_{1}}{a_{3}}\right)=f\left(\frac{a_{1}}{a_{2}}, \frac{a_{1}}{a_{3}}\right)-\frac{1}{\epsilon_{D D}},
$$

where the anisotropy function $f$ has to be evaluated separately for the three cases $a_{1}<a_{2}, a_{3}$ and $a_{2}<a_{1}, a_{3}$ as well as $a_{3}<a_{1}, a_{2}$. 


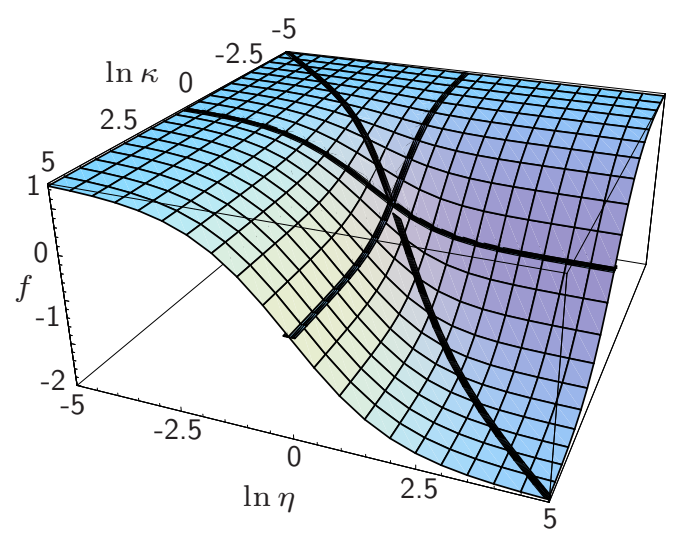

FIG. 2: Logarithmic plot of the anisotropy function $f(\eta, \kappa)$ in Eqs. (49) and (50). Black curves represent the special cases (53) with (54). The cross line corresponds to $f_{s}(\kappa)=f(\kappa, \kappa)$ and the lines parallel to the axes depict $f(1, \kappa)$ and $f(\kappa, 1)$.

\section{Anisotropy Function}

At first we consider the case $a_{1}<a_{2}, a_{3}$ and perform the substitution $\tau=\left(1 / a_{2}^{2}-1 / a_{3}^{2}\right) / z^{2}-1 / a_{2}^{2}$ which results in

$$
f(\eta, \kappa)=1+\frac{3 \kappa \eta}{\sqrt{1-\kappa^{2}}\left(1-\eta^{2}\right)}\left\{E\left(\arcsin \sqrt{1-\kappa^{2}}, \frac{\sqrt{1-\eta^{2}}}{\sqrt{1-\kappa^{2}}}\right)-F\left(\arcsin \sqrt{1-\kappa^{2}}, \frac{\sqrt{1-\eta^{2}}}{\sqrt{1-\kappa^{2}}}\right)\right\} \text { for } \kappa, \eta<1 .
$$

For the case $a_{3}<a_{2}<a_{1}$ we rather use the substitution $\tau=\left(1 / a_{1}^{2}-1 / a_{2}^{2}\right) / z^{2}-1 / a_{3}^{2}$, and yield

$$
\begin{aligned}
f(\eta, \kappa)= & \frac{1+2 \eta^{2}}{1-\eta^{2}}-\frac{3 \kappa \eta}{\left(1-\eta^{2}\right)\left(\kappa^{2}-1\right)}\left\{\sqrt{\kappa^{2}-\eta^{2}} E\left(\arcsin \frac{\sqrt{\kappa^{2}-\eta^{2}}}{\kappa}, \frac{\sqrt{\kappa^{2}-1}}{\sqrt{\kappa^{2}-\eta^{2}}}\right)\right. \\
& \left.-\frac{1-\eta^{2}}{\sqrt{\kappa^{2}-\eta^{2}}} F\left(\arcsin \frac{\sqrt{\kappa^{2}-\eta^{2}}}{\kappa}, \frac{\sqrt{\kappa^{2}-1}}{\sqrt{\kappa^{2}-\eta^{2}}}\right)\right\} \text { for } 1<\eta<\kappa .
\end{aligned}
$$

Finally, for the case $a_{2}<a_{3}<a_{1}$, we have a situation which is analogous to the second case when performing the replacement $\eta \leftrightarrow \kappa$ on the right hand side of (50). Note that all these different representations of the function $f$ are equivalent to each other by analytical continuation due to the property $[51,(8.127)]$. The importance of this anisotropy function for dipolar Bose gases has recently been recognized in the context of expanding chromium condensates [52]. There the anisotropy function appears within the Thomas-Fermi solution of the underlying Gross-Pitaevskii equation at zero temperature. Although our investigation of the critical temperature of dipolar gases represents a complete different physical situation, the anisotropy function plays here a similar important role. Figure 2 shows how the shape of this anisotropy function depends on its parameters $\eta$ and $\kappa$. For our further investigations we have to summarize the properties of $f(\eta, \kappa)$, see for comparison also Ref. [52]. At first, we mention its symmetry property

$$
f(\eta, \kappa)=f(\kappa, \eta)
$$

and, secondly, the sum rule

$$
f(\eta, \kappa)+f\left(\frac{\kappa}{\eta}, \frac{1}{\eta}\right)+f\left(\frac{1}{\kappa}, \frac{\eta}{\kappa}\right)=0 .
$$

Furthermore, we need the analytical continuation of this function into the semi-symmetric regions of the trap potential (3)

$$
f(\kappa, \kappa)=-2 f(1,1 / \kappa) \equiv f_{s}(\kappa)
$$


where the anisotropy function

$$
f_{s}(\kappa)=\left\{\begin{array}{cc}
\frac{2 \kappa^{2}+1}{1-\kappa^{2}}-\frac{3 \kappa^{2}}{\left(1-\kappa^{2}\right)^{3 / 2}} \operatorname{artanh} \sqrt{1-\kappa^{2}}, & 0<\kappa<1 \\
0 & \kappa=1 \\
\frac{2 \kappa^{2}+1}{1-\kappa^{2}}+\frac{3 \kappa^{2}}{\left(\kappa^{2}-1\right)^{3 / 2}} \arctan \sqrt{\kappa^{2}-1}, & \kappa>1
\end{array}\right.
$$

describes a cylindrically symmetric dipolar BEC Refs. [24-26, 53]. This function tends asymptotically to -2 and 1 in the limit $\kappa \rightarrow \infty$ and $\kappa \rightarrow 0$, respectively (see Figure 2).

\section{Results}

With the abbreviation (42) we find for our perturbative results (25), (26) and (34), (35) in the three extremal configurations $j=1,2,3$ of (44):

$$
\begin{aligned}
& \mathcal{F}^{(\mathrm{D})}=-\frac{\mu_{0} m^{2}}{6 \lambda^{3}(\hbar \beta \tilde{\omega})^{3}} I^{(j)}\left(\frac{\omega_{1}}{\omega_{2}}, \frac{\omega_{1}}{\omega_{3}}\right) \zeta_{\frac{3}{2}, \frac{3}{2}, \frac{3}{2}}\left(e^{\beta \mu}\right), \\
& \mathcal{F}^{(\mathrm{E})}=-\frac{\mu_{0} m^{2}}{6 \lambda^{3}(\hbar \beta \tilde{\omega})^{3}} I^{(j)}(1,1) \zeta_{\frac{3}{2}, \frac{3}{2}, \frac{3}{2}}\left(e^{\beta \mu}\right), \\
& \mu_{c}^{(\mathrm{D})}=-\frac{\mu_{0} m^{2}}{3 \lambda^{3}} I^{(j)}\left(\frac{\omega_{1}}{\omega_{2}}, \frac{\omega_{1}}{\omega_{3}}\right) \zeta\left(\frac{3}{2}\right), \\
& \mu_{c}^{(\mathrm{E})}=-\frac{\mu_{0} m^{2}}{3 \lambda^{3}} I^{(j)}(1,1) \zeta\left(\frac{3}{2}\right) .
\end{aligned}
$$

Here $\lambda$ denotes the thermodynamic de Broglie wave length

$$
\lambda=\sqrt{\frac{2 \pi \hbar^{2} \beta}{M}}
$$

and the generalization of the polylogarithmic function of Eq. (7) is defined according to

$$
\zeta_{a, b, c}(z)=\sum_{n=1}^{\infty} \sum_{n^{\prime}=1}^{\infty} \frac{z^{n+n^{\prime}}}{n^{a} n^{\prime b}\left(n+n^{\prime}\right)^{c}} .
$$

It is worth mentioning that both exchange terms of the grand-canonical energy (56) and the critical chemical potential (58) depend only on the contact interaction as the anisotropy function $f(1,1)$ vanishes in (48). Thus, within the semiclassical approach, only the direct contributions carrys the anisotropic character of the dipolar interaction.

\section{CRITICAL TEMPERATURE}

The total grand-canonical free energy in the three extremal configurations 1-3 of Figure 1 follows from (22) as the sum of (7), (55), and (56):

$$
\mathcal{F}=-\frac{1}{\beta(\hbar \beta \tilde{\omega})^{3}} \zeta_{4}\left(e^{\beta \mu}\right)-\frac{\mu_{0} m^{2}}{6 \lambda^{3}(\hbar \beta \tilde{\omega})^{3}}\left[I^{(j)}\left(\frac{\omega_{1}}{\omega_{2}}, \frac{\omega_{1}}{\omega_{3}}\right)+I^{(j)}(1,1)\right] \zeta_{\frac{3}{2}, \frac{3}{2}, \frac{3}{2}}\left(e^{\beta \mu}\right)+\ldots
$$

The full problem has to be studied at fixed particle number $N=-\partial \mathcal{F} / \partial \mu$ :

$$
N=\frac{1}{(\hbar \beta \tilde{\omega})^{3}} \zeta_{3}\left(e^{\beta \mu}\right)+\frac{\mu_{0} m^{2} \beta}{3 \lambda^{3}(\hbar \beta \tilde{\omega})^{3}}\left[I^{(j)}\left(\frac{\omega_{1}}{\omega_{2}}, \frac{\omega_{1}}{\omega_{3}}\right)+I^{(j)}(1,1)\right] \zeta_{\frac{1}{2}, \frac{3}{2}, \frac{3}{2}}\left(e^{\beta \mu}\right)+\ldots,
$$

where we have used the identity $\zeta_{\frac{3}{2}, \frac{3}{2}, \frac{1}{2}}(z)=2 \zeta_{\frac{1}{2}, \frac{3}{2}, \frac{3}{2}}(z)$ following from (60). The critical temperature $T_{c}$ is obtained from Eq. (62) in the limit $\mu \uparrow \mu_{c}=\mu_{c}^{(\mathrm{D})}+\mu_{c}^{(\mathrm{E})}+\ldots$. Recalling (57), (58), we obtain within first-order perturbation theory at the critical point

$$
N=\frac{1}{\left(\hbar \beta_{c} \tilde{\omega}\right)^{3}} \zeta(3)+\frac{\mu_{0} m^{2} \beta_{c}}{3 \lambda_{c}^{3}\left(\hbar \beta_{c} \tilde{\omega}\right)^{3}}\left[I^{(j)}\left(\frac{\omega_{1}}{\omega_{2}}, \frac{\omega_{1}}{\omega_{3}}\right)+I^{(j)}(1,1)\right]\left\{\zeta\left(\frac{1}{2}, \frac{3}{2}, \frac{3}{2}\right)-\zeta\left(\frac{3}{2}\right) \zeta(2)\right\}+\ldots,
$$


where $\zeta(a, b, c)=\zeta_{a, b, c}(1)$ is a generalization of Riemanns zeta-function. The first term in Eq. (63) reproduces the interaction-free particle number, thus leading to the critical temperature (15). The interaction term changes this, so that the resulting shift of the critical temperature in configurations $1-3$ reads according to (48), (53), and (54)

$$
\begin{aligned}
& \left(\frac{\Delta T_{c}}{T_{c}^{(0)}}\right)^{(1)}=-c_{\delta} \frac{a}{\lambda_{c}^{(0)}}+\frac{c_{\delta}}{2} \frac{\mu_{0} m^{2} M}{12 \pi \hbar^{2} \lambda_{c}^{(0)}} f\left(\frac{\omega_{1}}{\omega_{2}}, \frac{\omega_{1}}{\omega_{3}}\right), \\
& \left(\frac{\Delta T_{c}}{T_{c}^{(0)}}\right)^{(2)}=-c_{\delta} \frac{a}{\lambda_{c}^{(0)}}+\frac{c_{\delta}}{2} \frac{\mu_{0} m^{2} M}{12 \pi \hbar^{2} \lambda_{c}^{(0)}} f\left(\frac{\omega_{2}}{\omega_{3}}, \frac{\omega_{2}}{\omega_{1}}\right), \\
& \left(\frac{\Delta T_{c}}{T_{c}^{(0)}}\right)^{(3)}=-c_{\delta} \frac{a}{\lambda_{c}^{(0)}}+\frac{c_{\delta}}{2} \frac{\mu_{0} m^{2} M}{12 \pi \hbar^{2} \lambda_{c}^{(0)}} f\left(\frac{\omega_{3}}{\omega_{1}}, \frac{\omega_{3}}{\omega_{2}}\right) .
\end{aligned}
$$

The dimensionless prefactor $c_{\delta}$ for the contact interaction has the value

$$
c_{\delta}=\frac{4}{3 \zeta(3)}\left[\zeta\left(\frac{3}{2}\right) \zeta(2)-\zeta\left(\frac{1}{2}, \frac{3}{2}, \frac{3}{2}\right)\right] \approx 3.426 .
$$

Now we discuss the physical implications of our first-order perturbative result (64). By setting $m=0$ we obtain for all configurations 1-3 in Figure 1 the same downwards shift of the critical temperature with the dimensionless prefactor (65). This simple result for the isotropic contact interaction was originally derived in Ref. [28] within a mean-field approach. Only recently it was confirmed experimentally by investigating the onset of Bose-Einstein condensation in the hyperfine ground state of ${ }^{87} \mathrm{Rb}$ [54]. Furthermore, our result (64) also shows how the shift of the critical temperature depends on the anisotropic magnetic dipole-dipole interaction.

So far we restricted our detailed analysis to those spherical angles $(\alpha, \phi)$ between the symmetry axes of the interaction (2) and the trap (3) which coincide with the extremal cases (44). It is straight-forward to extend our result to arbitrary angles. For this purpose we use the master integral (41) and its decomposition (43) into the integral (42) for $j=1,2,3$. With this we calculate the following angle-dependent shift of the critical temperature:

$$
\left(\frac{\Delta T_{c}}{T_{c}^{(0)}}\right)^{(\alpha, \phi)}=-c_{\delta} \frac{a}{\lambda_{c}^{(0)}}+\frac{c_{\delta}}{2} \frac{\mu_{0} m^{2} M}{12 \pi \hbar^{2} \lambda_{c}^{(0)}}\left\{\sin ^{2} \alpha\left[\cos ^{2} \phi f\left(\frac{\omega_{1}}{\omega_{2}}, \frac{\omega_{1}}{\omega_{3}}\right)+\sin ^{2} \phi f\left(\frac{\omega_{2}}{\omega_{3}}, \frac{\omega_{2}}{\omega_{1}}\right)\right]+\cos ^{2} \alpha f\left(\frac{\omega_{3}}{\omega_{1}}, \frac{\omega_{3}}{\omega_{2}}\right)\right\} .
$$

For the special cases of $\alpha=0, \alpha=\pi / 2$ and $\phi=0, \pi / 2, \pi, 3 \pi / 2$, Eq. (66) reduces to the previous results (64), which turn out to be extremal.

\section{MAGIC ANGLES}

Changing the angles $(\alpha, \phi)$ between the symmetry axes of the trap and the magnetization direction allows us to tune the dipolar effect between those maximal and minimal values. In particular, there exist so-called magic angles, where the dipolar effects vanish. We read off from Eq. (66) that those angles are given for an arbitrary anisotropy due to the sum rule (52) by

$$
\alpha_{0}(\phi)=\operatorname{arccot}\left\{ \pm \sqrt{\sin ^{2} \phi-\cos 2 \phi f\left(\frac{\omega_{1}}{\omega_{2}}, \frac{\omega_{1}}{\omega_{3}}\right) / f\left(\frac{\omega_{3}}{\omega_{1}}, \frac{\omega_{3}}{\omega_{2}}\right)}\right\} .
$$

Note that the radicand has to be positive, which restricts the range of values for the polar angles $\phi$. In general, the possibility to find a magic angle depends crucially on the anisotropy coefficients. Only in the case that $f\left(\omega_{3} / \omega_{1}, \omega_{3} / \omega_{2}\right)<-f\left(\omega_{1} / \omega_{2}, \omega_{1} / \omega_{3}\right)$ is fulfilled, magic angles always exist.

Now we present the results for cylindrically symmetric traps. At first, we mention for $\omega_{1}=\omega_{2}$ that the magic angle (67) reduces to a value, which is independent of the second anisotropy parameter $\omega_{1} / \omega_{3}$. This value is also $\phi$-independent according to (67) with (53) and amounts to $\alpha_{0}=\operatorname{arccot}( \pm 1 / \sqrt{2})=54.7^{\circ}$ or $125.3^{\circ}[20,26]$. For another symmetric case with $\omega_{1}=\omega_{3}$ we find with (53) that $f\left(\omega_{3} / \omega_{1}, \omega_{3} / \omega_{2}\right)=f\left(\omega_{1} / \omega_{2}, \omega_{1} / \omega_{3}\right)$, thus solutions of (67) only exist for $\sin ^{2} \phi>1 / 3$. The locations of magic spherical angles for both symmetric cases $\omega_{1}=\omega_{2}$ and $\omega_{1}=\omega_{3}$ are depicted in Figure 3 a). For the third symmetric case $\omega_{2}=\omega_{3}$ the situation is similar to $\omega_{1}=\omega_{3}$ as the polar angle $\phi$ is only shifted by $\pi / 2$. 
a)

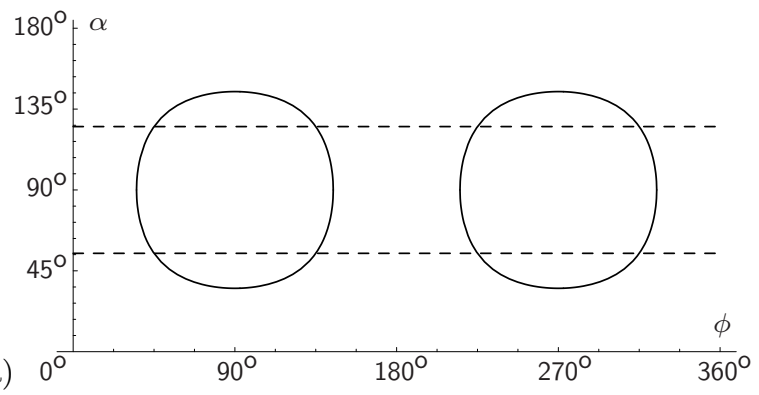

b)

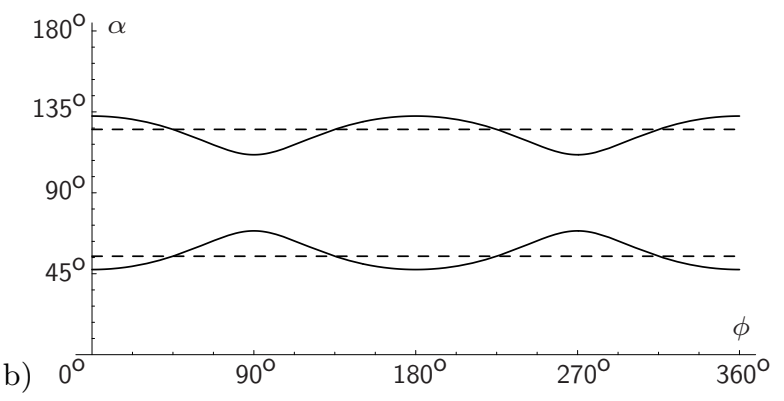

FIG. 3: The location of magic angles $(\alpha, \phi)$, where the dipolar effect vanishes. a) The solid line stands for a cylinder symmetric problem with $\omega_{1}=\omega_{3}$ and the dashed line for that with symmetry $\omega_{1}=\omega_{2}$. b) The solid line stands for the anisotropic parameters of the Stuttgart experiment and the dashed line for a cylinder symmetric problem with $\omega_{1}=\omega_{2}$.

Furthermore, we discuss the situation in a complete anisotropic harmonic trap by the example of the trap frequencies of the Stuttgart experiment [5] which are given by $\omega_{1}=2 \pi \cdot 581 \mathrm{~Hz}, \omega_{2}=2 \pi \cdot 406 \mathrm{~Hz}$, and $\omega_{3}=2 \pi \cdot 138 \mathrm{~Hz}$. Thus, we find from $(50)$ the values $f\left(\omega_{1} / \omega_{2}, \omega_{1} / \omega_{3}\right)=-0.6252$ and $f\left(\omega_{3} / \omega_{1}, \omega_{3} / \omega_{2}\right)=+0.7356$. Specializing the general result (67) for the Stuttgart experiment leads to

$$
\alpha_{0}(\phi)=\operatorname{arccot}\left\{ \pm \sqrt{0.150 \sin ^{2} \phi+0.850 \cos ^{2} \phi}\right\} .
$$

This behaviour is depicted in Figure $3 \mathrm{~b}$ ), where we see the location of the magic angles compared with the $\phi$ independent value of a problem with a cylinder symmetry around the $z$-axis.

\section{STUTTGART EXPERIMENT}

Here we discuss the consequences of our results for the concrete example of the parameters from the ongoing experiments on the Bose-Einstein condensation of ${ }^{52} \mathrm{Cr}$-atoms at the University of Stuttgart [5]. There the geometric mean frequency is $\tilde{\omega}=2 \pi \cdot 319 \mathrm{~Hz}$. The total atom number is $N=100000$, yielding an interaction-free critical temperature (15) of about $T_{c}^{(0)}=670 \mathrm{nK}$. The finite-size correction of $T_{c}^{(0)}$ in the Stuttgart experiment follows from (16) and amounts to $-1.8 \%$. This can now be compared with a shift of the critical temperature due to the contact and the magnetic dipole-dipole interaction.

The scattering length of the ${ }^{52} \mathrm{Cr}$-atoms is given by $a \approx 105 a_{B}$ with the Bohr radius $a_{B}$ [55], so we obtain from (38) $\epsilon_{D D}=0.144$. The thermodynamic de Broglie wave length has the value $\lambda_{c}^{(0)} \approx 5598 a_{B}$ for the parameter of Stuttgart experiment. From Eq. (64) we obtain from the contact interaction a downwards shift of the critical temperature by $-6.4 \%$. This is now modified by the magnetic dipole-dipole interaction. At first, we consider case 1 from Figure 1, in which the magnetization is parallel to the trap axes with the largest moment of inertia. There we find from (50) the result $f\left(\omega_{1} / \omega_{2}, \omega_{1} / \omega_{3}\right)=-0.6252$, which leads via Eq. (64) to an extra downward shift of the critical temperature by $-0.29 \%$ due to the magnetic dipole-dipole interaction. For the case 2 of Figure 1 we find $f\left(\omega_{2} / \omega_{3}, \omega_{2} / \omega_{1}\right)=-0.1104$, which amounts to an additional downward shift of the critical temperature due to the magnetic dipole-dipole interaction by $-0.05 \%$. Finally, for case 3 of Figure 1 with the magnetization direction parallel to the trap axes with the smallest moment of inertia, we find $f\left(\omega_{3} / \omega_{1}, \omega_{3} / \omega_{2}\right)=+0.7356$ which results in an upward shift of the critical temperature due to the dipole-dipole interaction of about $+0.34 \%$. Figure $4 \mathrm{a}$ ) shows the resulting total shift of the critical temperature $\Delta T_{c}$ for the ${ }^{52} \mathrm{Cr}$ gas with respect to the interaction-free critical temperature $T_{c}^{(0)}$ versus the particle number $N$. Both the finite-size corrections and the contact interaction lead to a huge shift, on top of which the small dipolar effect is seen.

Furthermore, we we follow Ref. [26] and suggest to measure differences between the critical temperatures in the three extremal cases of Figure 1. The large shift caused by the finite-size corrections (16) and the contact interaction would cancel due to their isotropic character. Thus, the difference between the shifts is exclusively caused by the magnetic dipole-dipole interaction. For the total atom number $N=100000$ and the mean frequency $\tilde{\omega}=2 \pi \cdot 319 \mathrm{~Hz}$, the temperature difference between the case 1 and 3 amounts to a net effect of $0.61 \%$ of the interaction-free critical 

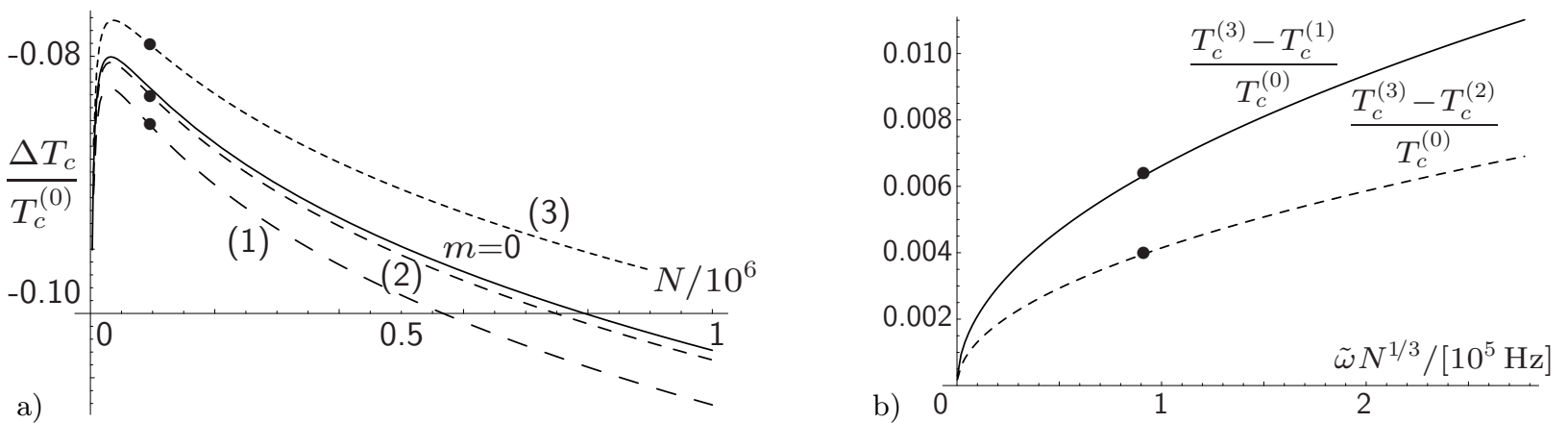

FIG. 4: a) Shift of the critical temperature $\Delta T_{c}$ with respect to the interaction-free critical temperature $T_{c}^{(0)}$ for a ${ }^{52} \mathrm{Cr}$ gas in a harmonic trap with frequencies $\omega_{1}=2 \pi \cdot 581 \mathrm{~Hz}, \omega_{2}=2 \pi \cdot 406 \mathrm{~Hz}$, and $\omega_{3}=2 \pi \cdot 138 \mathrm{~Hz}$ calculated from (64) and (16) without (straight line) and with (dashed lines) magnetic dipole-dipole interaction for the cases 1-3 of Figure 1. b) Differences of the critical temperature shifts versus effective parameter $\tilde{\omega} N^{1 / 3}$. The respective dots indicate the present parameters of the Stuttgart ${ }^{52} \mathrm{Cr}$ experiment.
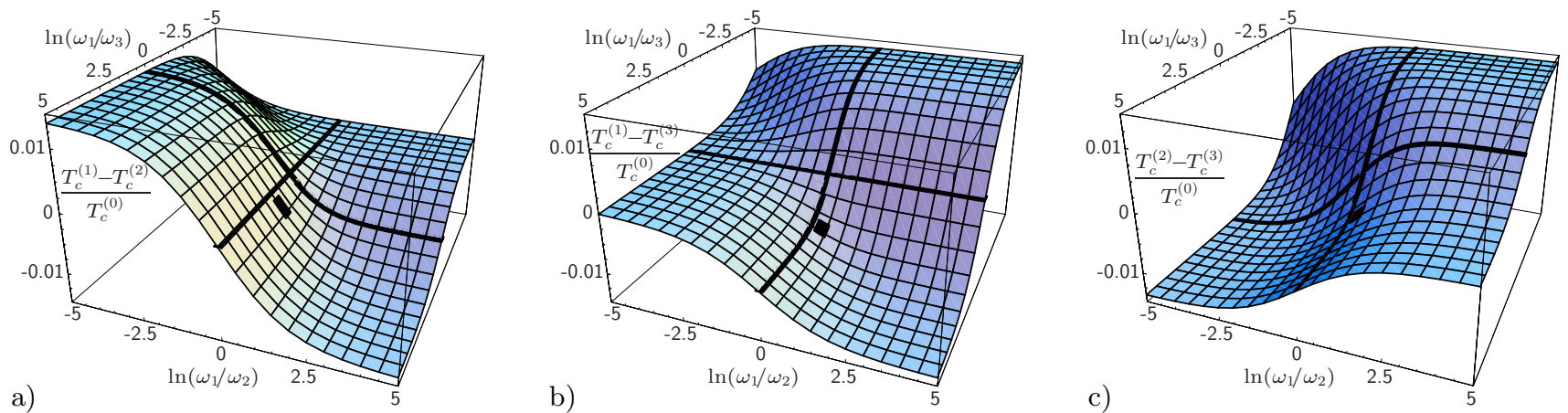

FIG. 5: Shifts of critical temperatures a) $\Delta T_{c}^{(1)}-\Delta T_{c}^{(2)}$, b) $\Delta T_{c}^{(1)}-\Delta T_{c}^{(3)}$ and c) $\Delta T_{c}^{(2)}-\Delta T_{c}^{(3)}$ with respect to the interaction-free critical temperature $T_{c}^{(0)}$ for $N=10^{5}{ }^{52} \mathrm{Cr}$-atoms in a trap with mean frequency $\tilde{\omega}=2 \pi \cdot 319 \mathrm{~Hz}$ versus anisotropy parameters $\omega_{1} / \omega_{2}$ and $\omega_{1} / \omega_{3}$ according to (64). The black curves represent cylindrically symmetric cases and the black squares the values of the Stuttgart experiment.

temperature $T_{c}^{(0)}$. This dipolar effect increases with the geometric mean frequency $\tilde{\omega}$ and the total atom number $N$ as $\left[\tilde{\omega} N^{1 / 3}\right]^{1 / 2}$, as is shown in Figure $4 \mathrm{~b}$ ). Another possibility to enhance the difference of the critical temperatures is provided by varying the anisotropy strengths $\omega_{1} / \omega_{2}$ and $\omega_{1} / \omega_{3}$ of the harmonic trap potential as seen in Figure 5 . For instance, in order to increase the difference of the critical temperature between the first and third configuration, we need to increase the aspect ratio $\omega_{1} / \omega_{2}$. Furthermore, it is preferable to work with moderate anisotropy parameters $\omega_{1} / \omega_{3}<1$ (pancake like) than in the regime $\omega_{1} / \omega_{3}>1$ (cigar shaped) of the Stuttgart experiment, as is indicated by Figure $5 \mathrm{~b})$.

Finally, we elucidate how to achieve measurable dipolar effects at the critical point. At first, varying the anisotropy parameters only allows to increase the critical temperature difference $T_{c}^{(3)}-T_{c}^{(1)}$ with respect to the interaction-free value from currently $0.61 \%$ up to a maximal value of around $1.39 \%$. Note that the latter represents an upper limit according to our perturbative calculations. Not so strictly limited is the possibility to amplify the magnetic dipolar effect by increasing the particle number or the mean trap frequency. For instance, a doubling of the latter yields by constant particle number an increase of the effect by the factor 1.41. An even higher increase of the particle number seems to be promising, but is in fact physically limited by three-body losses.

\section{CONCLUSION}

So far, the dipolar nature of nowdays available BECs has only been resolved in expansion experiments [21]. The analysis of the present article shows that it could become possible to detect a signal of the underlying dipole-dipole 
interaction also from measuring the critical temperature of the onset of Bose-Einstein condensation. Most sensitive is the difference of the critical temperatures where the magnetization direction is parallel to the axes of the harmonic trap with largest and smallest moment of inertia. We have shown quantitatively that this temperature difference increases with the number of chromium atoms, the geometrical mean frequency, and the anisotropy of the trap. Furthermore, our results will, certainly, be useful also for other dipolar systems with a tunable dipole moment, like heteronuclear molecules in low vibrational states $[11-13,15-18]$, where the dipolar effect will be larger.

\section{Acknowledgement}

It is a pleasure to thank Hagen Kleinert, Tilman Pfau, and Shai Ronen for stimulating discussions. Furthermore, we acknowledge financial support from the DFG Priority Program SPP 1116 Interactions in Ultra-Cold Atomic and Molecular Gases. 


\section{References}

[1]M.H. Anderson, J.R. Ensher, M.R. Matthews, C.E. Wieman, and E.A. Cornell, Science 269, 198 (1995)

[2]K.B. Davis, M.-O. Mewes, M.R. Andrews, N.J. van Druten, D.S. Durfee, D.M. Kurn, and W. Ketterle, Phys. Rev. Lett. 75, 3969 (1995)

[3]M. Greiner, O. Mandel, T. Esslinger, T.W. Hänsch, and I. Bloch, Nature 415, 39 (2002)

[4]S. Inouye, M.R. Andrews, J. Stenger, H.-J. Miesner, D.M. Stamper-Kurn, and W. Ketterle, Nature 392, 151 (1998)

[5]A. Griesmaier, J. Werner, S. Hensler, J. Stuhler and T. Pfau, Phys. Rev. Lett. 94, 160401 (2005)

[6]L. Santos, G.V. Shlyapnikov, P. Zoller, and M. Lewenstein, Phys. Rev. Lett. 85, 1791 (2000)

[7]K. Afrousheh, P. Bohlouli-Zanjani, J.D. Carter, A. Mugford, and J.D.D. Martin, Phys. Rev. A 73, 063403 (2006)

[8]S. Yi and L. You, Phys. Rev. A 63, 053607 (2000)

[9]K. Góral, K. Rzazewski, and T. Pfau, Phys. Rev. A 61, 051601(R) (2000)

[10]J.P. Martikainen, M. Mackie, and K.A. Suominen, Phys. Rev. A 64, 037601 (2001)

[11]J.D. Weinstein, R. de Carvalho, T. Guillet, B. Friedrich, and J.M. Doyle, Nature 395, 148 (1998)

[12]J.M. Doyle and B. Friedrich, Nature 401, 749 (1999)

[13]H.L. Bethlem, G. Berden, and G. Meijer, Phys. Rev. Lett. 83, 1558 (1999)

[14]S.Y.T. van de Meerakker, P.H.M. Smeets, N. Vanhaecke, R.T. Jongma, and G. Meijer, Phys. Rev. Lett. 94, 023004 $(2005)$

[15]J.M. Sage, S. Sainis, T. Bergeman, and D. DeMille, Phys. Rev. Lett. 94, 203001 (2005)

[16]D. Wang, J. Qi, M.F. Stone, O. Nikolayeva, B. Hattaway, S.D. Gensemer, H. Wang, W.T. Zemke, P.L. Gould, E.E. Eyler, and W.C. Stwalley, Euro. Phys. J. D 31, 165 (2004)

[17]M.W. Mancini, G.D. Telles, A.R.L. Caires, V.S. Bagnato, and L.G. Marcassa, Phys. Rev. Lett. 92, 133203 (2004)

[18]C. Ospelkaus, S. Ospelkaus, L. Humbert, P. Ernst, K. Sengstock, and K. Bongs, Phys. Rev. Lett. 97, 120402 (2006)

[19]J.D. Jackson, Classical Electrodynamics, Second Edition (John Wiley \& Sons, New York, 1975)

[20]S. Giovanazzi, A. Görlitz, and T. Pfau, Phys. Rev. Lett. 89, 130401 (2002)

[21]J. Stuhler, A. Griesmaier, T. Koch, M. Fattori, T. Pfau, S. Giovanazzi, P. Pedri, and L. Santos, Phys. Rev. Lett. 95, $150406(2005)$

[22]L. Santos, G.V. Shlyapnikov, and M. Lewenstein, Phys. Rev. Lett. 90, 250403 (2002)

[23]K. Góral and L. Santos, Phys. Rev. A 66, 023613 (2002)

[24]D.H.J. O’Dell, S. Giovanazzi, and C. Eberlein, Phys. Rev. Lett. 92, 250401 (2004)

[25]C. Eberlein, S. Giovanazzi, and D.H.J. O’Dell, Phys. Rev. A 71, 033618 (2005)

[26]K. Glaum, A. Pelster, H. Kleinert, and T. Pfau, Phys. Rev. Lett. 98, 080407 (2007)

[27]B. Kastening, Phys. Rev. A 70, 043621 (2004)

[28]S. Giorgini, L.P. Pitaevskii, and S. Stringari, Phys. Rev. A 54, R4633 (1996)

[29]H. Kleinert, Path Integrals in Quantum Mechanics, Statistics, Polymer Physics, and Financial Markets, Forth Edition (World Scientific, Singapore, 2006)

[30]H. Kleinert and V. Schulte-Frohlinde, Critical Properties of $\phi^{4}$-Theories (World Scientific, Singapore, 2001) 
[31]S. Kling and A. Pelster, Phys. Rev. A (in press), eprint: cond-mat/0604162

[32]S. Grossmann and M. Holthaus, Phys. Lett. A 208, 188 (1995)

[33]T. Haugset, H. Haugerud, and J.O. Andersen, Phys. Rev. A 55, 2922 (1997)

[34]F. Dalfovo, S. Giorgini, L.P. Pitaevskii, and S. Stringari, Rev. Mod. Phys. 71, 463 (1999)

[35]Ph.W. Courteille, V.S. Bagnato, and V.I. Yukalov, Laser Phys. 11, 659 (2001)

[36]K. Huang, Statistical Mechanics (John Wiley \& Sons, New York, 1963)

[37]A.A. Abrikosov, L.P. Gorkov, and I.E. Dzyaloshinski, Methods of Quantum Field Theory in Statistical Physics (Pergamon Press, New York, 1965)

[38]A.L. Fetter and J.D. Walecka, Quantum Theory of Many-Particle Systems (McGraw-Hill, New York, 1971)

[39]J.W. Negele and H. Orland, Quantum Many-Particle Systems (Addison-Wesley, New York, 1988)

[40]E.K.U. Gross, E. Runge, and O. Heinonen, Many Particle Theory (Adam Hilger, Bristol, 1991)

[41]G.D. Mahan, Many-Particle Physics, Third Edition (Kluwer Academic/Plenum Publisher, New York, 2000)

[42]A. Pelster and K. Glaum, Phys. Stat. Sol. B 237, 72 (2003)

[43]A. Pelster, H. Kleinert, and M. Bachmann, Ann. Phys. (N.Y.) 297, 363 (2002)

[44]A. Pelster and K. Glaum, Physica A 335, 455 (2004)

[45]D.C.E. Bortolotti, S. Ronen, J.L. Bohn, and D. Blume, Phys. Rev. Lett. 97, 160402 (2006)

[46]S. Ronen, D.C.E. Bortolotti, D. Blume, and J.L. Bohn, Phys. Rev. A 74, 033611 (2006)

[47]K. Huang and C.N. Yang, Phy. Rev. 105, 767 (1957)

[48]A. Derevianko, Phys. Rev. A 67, 033607 (2003); Phys. Rev. A 72, 039901(E) (2005)

[49]S. Yi and L. You, Phy. Rev. Lett 92, 193201 (2004)

[50]C. Cohen-Tannoudji, J. Dupont-Roc, and G. Grynberg, Photons and Atoms - Introduction to Quantum Electrodynamics (John Wiley \& Sons, New York, 1989)

[51]I.S. Gradshteyn and I.M. Ryzhik, Table of Integrals, Series, and Products (Academic Press, New York, 1965)

[52]S. Giovanazzi, P. Pedri, L. Santos, A. Griesmaier, M. Fattori, T. Koch, J. Stuhler, and T. Pfau, Phys. Rev. A 74, $013621(2006)$

[53]S. Giovanazzi, A. Görlitz, and T. Pfau, J. Opt. B 5, S208 (2003)

[54]F. Gerbier, J.H. Thywissen, S. Richard, M. Hugbart, P. Bouyer, and A. Aspect, Phys. Rev. Lett. 92, 030405 (2004)

[55]J. Werner, A. Griesmaier, S. Hensler, J. Stuhler, T. Pfau, A. Simoni, and E. Tiesinga, Phys. Rev. Lett. 94, 183201 (2005) 